\title{
Substantial Nation-Wide Improvement in Lung Cancer Relative Survival in Norway from 2000 to 2016
}

Odd Terje Brustugun, MD PhD, ${ }^{1 *}$ Bjørn Henning Grønberg, MD PhD, ${ }^{2,3}$ Lars Fjellbirkeland, MD PhD, ${ }^{4,5}$ Nina Helbekkmo, MD PhD, ${ }^{6}$ Marianne Aanerud, MD PhD, ${ }^{7}$ Tom Kristian Grimsrud, MD PhD, ${ }^{8}$ Åslaug Helland, MD PhD, ${ }^{5,9}$ Bjørn Møller, PhD, ${ }^{8}$ Yngvar Nilssen, PhD, ${ }^{8}$ Trond Eirik Strand, MD PhD, ${ }^{8}$ Steinar Kristian Solberg, MD PhD ${ }^{10}$

${ }^{1}$ Section of Oncology, Drammen Hospital, Vestre Viken Hospital Trust, Drammen, Norway

${ }^{2}$ The Cancer Clinic, St. Olav's Hospital, Trondheim University Hospital, Trondheim, Norway

${ }^{3}$ Faculty of Medicine and Health Sciences, Department of Clinical and Molecular Medicine, NTNU, Norwegian University of Science and Technology, Trondheim, Norway

${ }^{4}$ Dept of Pulmonology, Oslo University Hospital, Oslo, Norway

${ }^{5}$ Faculty of Medicine, University of Oslo, Oslo, Norway

${ }^{6}$ Dept of Pulmonology and Dept of Oncology, University Hospital of North Norway, Troms $\varnothing$, Norway

${ }^{7}$ Dept of Pulmonology, Haukeland University Hospital, Bergen, Norway

${ }^{8}$ Cancer Registry of Norway, Oslo, Norway

${ }^{9}$ Dept of Oncology, Oslo University Hospital, Oslo, Norway

${ }^{10}$ Dept of Thoracic Surgery, Oslo University Hospital, Oslo, Norway

*Corresponding author:

Odd Terje Brustugun, Section of Oncology, Drammen Hospital - Vestre Viken Hospital Trust, PO Box 800, N-3004 Drammen, Norway

Email: otr@vestreviken.no

Text word count: 3134 words

Abstract word count: 247 words

Number of figures/tables: 6

Number of references: 30 


\section{Abstract}

\section{Introduction}

There have been significant changes in both diagnostic procedures and therapy for lung cancer since the beginning of the millennium. National incidence and survival data from 2000 through 2016 are studied.

\section{Methods}

The national registry data are virtually complete. For survival analysis, the period analysis method was applied.

\section{Results}

A total of 44,825 individuals were diagnosed with lung cancer in Norway in the study period. The number of incident cases increased with $49 \%$ whereas the prevalence increased with $136 \%$ from 2000 to 2016 . In 2016, adenocarcinoma accounted for $46 \%$ and $52 \%$ of lung cancers in males and females respectively. The entity "NSCLC not otherwise specified" declined from $24 \%$ to $13 \%$ and the fraction of patients with metastatic disease decreased from $54 \%$ to $46 \%$ during the period, for both sexes combined.

The overall median survival more than doubled from 6.5 to 14.3 months for females and from 5.4 to 11.4 months in males. Median survival for patients with metastatic disease increased from 3.6 to 5.1 months among females and from 3.2 to 4.2 months among males. For non-surgically treated cases with localised disease, 5 -year survival increased from $25 \%$ to more than $40 \%$ in females, and from $10 \%$ to almost $40 \%$ in males.

\section{Conclusion}

There have been notable changes in incidence patterns and a remarkable improvement in survival for lung cancer over the last 16 years, though the gain of survival in patients with metastatic disease was 
modest. Hopefully, this subgroup of patients will have significantly bettered outcomes as immunotherapy is implemented.

Key words

Lung cancer

Epidemiology

Survival

Incidence

Registry 


\section{Introduction}

Lung cancer is the most frequent cause of cancer-related death in developed countries, responsible for $5.3 \%$ of all deaths and $22 \%$ of all cancer deaths in Norway [1-3]. It is increasingly clear that the various morphological entities of lung cancer are biologically distinct, and may have differences both in incidence patterns, stage distribution, as well as prognosis. Furthermore, these characteristics are changing, possibly due to changes in smoking patterns and the composition of cigarettes [4]. Notably, there are major differences in incidence and survival between the sexes [5].

There have been significant changes in both diagnostic procedures and lung cancer therapy since the beginning of the millennium. This has improved the prognosis for patients with lung cancer, but also increased the costs and requirement for resources of the health care services [6].

The years from the turn of the millennium has been a period when modern procedures for diagnosis and staging of lung cancer became generally available, and stereotactic body radiotherapy (SBRT) and new systemic therapies were introduced. Chemotherapy was widely applied from 2000 [7], PET-CT for staging has been available since 2006 [8], stereotactic body radiotherapy (SBRT) was introduced at the national level from 2008 [9], and mutational analyses were implemented from 2010 [10]. Immunotherapy is believed to have a significant impact on the prognosis of lung cancer, but until 2016, Norwegian patients did not receive immunotherapy, apart from a small number who participated in clinical studies or compassionate use programs [11]. It is of great interest to have comprehensive base-line data on patients with lung cancer and treatment outcomes before implementing such therapy. Thus, the aims of this study were to investigate trends in incidence and survival data on a national level for lung cancer cases diagnosed from 2000 to 2016 


\section{Materials and methods}

\section{Population demographics}

The population of Norway increased from 4.5 mill in 2000 to 5.2 mill in 2016 (16.4\% growth). The fraction of people aged 67 years or older was 13.8\% in 2000 and 14.3\% in 2016 (Statistics Norway, ssb.no). Norway provides uniform and public health care services financed by taxation and a national insurance system for all residents, independent of social status. Still, some socioeconomic and geographical differences in health and health care exist [12], but these were disregarded under the assumption that they only marginally influence the overall national trends in incidence and survival [13]. Furthermore, the vast majority of patients are Caucasians. Thus, subset analyses based on socioeconomic status, geography of ethnicity were not conducted.

\section{Data collection}

All cases of lung cancer, ICD-10 C34, diagnosed in the period 2000-2016 and reported to the Cancer Registry of Norway (CRN) were included. Cases with death certificate only and unknown histology were also included in the study group.

Since 1952 it has been mandatory by law to report all malignant neoplasms to the CRN. In addition, copies of cytology, biopsy, and autopsy reports from all pathology laboratories as well as death certificate reports from the Cause of Death Registry run by Norwegian Institute of Public Health, have routinely been sent to the CRN. Since 1998, all hospitals have filed discharge summaries electronically to the registry for patients with cancer diagnosis. The system for reporting to the CRN was evaluated in 2009 , and overall completeness was estimated to be $98.8 \%$ [14].

Baseline data in CRN in addition to diagnosis and time of diagnosis is age, sex, condensed stage, morphology where known and treatment with surgery or radiotherapy. From 2015 lung cancer reporting 
to CRN was extended through electronically reporting forms with more extensive information on every case such as detailed TNM, performance status and more.

The CRN receive monthly updates from Statistics Norway on vital status (death, or emigration) including date of occurrence. All doctors are required by law to complete a death certificate which is submitted to Norwegian Cause of Death Registry and the cause of death is coded according to International Classification of Diseases, 10th revision (ICD-10). From 2005 the Automated Classification of Medical Entities (ACME) system was implemented.

During the study period (2000-2016) procedures for diagnosis and staging were assumed to be uniform throughout the country. Cases notified to the cancer registry by death certificate only and cases who were diagnosed post mortem were excluded from the survival analyses as survival time for these cases would effectively be zero.

Data on the use of SBRT for lung cancer were collected from the department of medical physics from the four Norwegian university hospitals delivering such therapy.

\section{Diagnosis and staging}

The CRN utilises a condensed staging system for lung cancer reporting, based on the TNM classification system, in that T1-2N0M0 is defined as 'Localised' (stage I), M1 as 'Metastatic' (stage IV) and the others as 'Regionally advanced' (stages II and III). Tumour localisation was coded according to ICD-10, and tumour histology was coded according to the Manual of Tumor Nomenclature and Coding. Since 1993, both topography and morphology have been coded according to the International Classification of Diseases for Oncology (ICD-O). 


\section{Patient follow-up}

In survival analyses, the primary outcome was survival following a diagnosis of lung cancer. Follow-up was defined as the time from lung cancer diagnosis to death or emigration as reported from Statistics Norway or up to December 312016 for patients who were still alive at that date.

\section{Statistical methods}

The CRN database November 2017 submission was used for all analyses. In addition to sex, surgery and histological type, demographic characteristics including age at diagnosis and stage of disease were evaluated. The study period was divided into calendar years.

Incidence and prevalence

One- and 5-year male and female relative survival (RS) stratified by age, stage, surgery and histological type was calculated for individuals diagnosed through successive 5-year calendar periods. RS for men and women was estimated using the method of Perme et al [15]. RS adjusts for competing causes of death expected for persons of the same sex, age, and calendar year of investigation (the ratio of observed survival in the cancer population to the expected survival rate in a comparable cancer-free population); hence information about specific cause of death is not required.

For survival analysis, the period analysis method including the survival experience in the most recent calendar years was applied, ensuring that up-to-date estimates of long-term survival could be provided in a situation with improvement of survival over time [16].

The European standard population (ESP2013) from Eurostat was used as reference for age adjustment of death rates. 


\section{Results}

\section{Incidence}

\section{Demography}

44,825 new lung cancer cases were diagnosed during the period. In total, $57 \%$ of cases were males, but the fraction of females increased throughout the period, from 38\% in 2000 to $48 \%$ in 2016 (Fig 1A). In absolute numbers there was a near doubling in females from $7 x x$ to $15 x x$ cases.

Both the number of incident cases and the prevalence increased substantially during the study period, with an average annual incidence increase of $4.2 \%$ among females and $1.4 \%$ among males. The yearly rate of increase for females in the first 5 -year period was $4.0 \%$, and $3.7 \%$ in the last 5 -year period. Corresponding numbers for males were $1.8 \%$ and $-0.3 \%$, and for both sexes combined $2.7 \%$ and $1.5 \%$.

The prevalence of individuals alive after a lung cancer diagnosis more than doubled from the end of 2000 until the end of 2016, from 3185 to 7507 (136\% increase) (Fig 1B). The prevalence of subjects surviving a cancer diagnosis (all types combined) increased with 76\% in the same period (data not shown). In 2016, $66 \%$ of patients with lung cancer had lived less than five years after they were diagnosed, while the corresponding number for all patients with cancer was $37 \%$ (data not shown). Thus most of the lung cancer survivors are in active treatment or surveillance programs, in contrast to cancer patients in general.

The mean age at diagnosis was lower for females than males throughout the period, but the difference was diminishing. In 2000, the mean age at diagnosis was 69.3 and 67.0 for males and females respectively. After 16 years, the mean age had increased with 2 years for men and 3.7 years for women thus being close to 71 years for both sexes (Fig 1C). 


\section{Histology}

The average annual increase in female number of cases diagnosed with adenocarcinoma was $6.6 \%$ during the period, and $4.5 \%$ for squamous cell carcinoma. For men, the annual increase was $4.8 \%$ for adenocarcinoma and $0.5 \%$ for squamous cell carcinoma. Adenocarcinoma was the most common subtype among females during the whole period, and surpassed squamous cell carcinoma as the most frequent subtype also among males in the beginning of the study period. In 2016, adenocarcinoma was the most prevalent histology subtype accounting for $46 \%$ of lung cancers in males, and $52 \%$ in females (Fig 2).

In addition, diagnostic entities other than small cell lung carcinoma (SCLC), squamous cell carcinomas and adenocarcinomas (typically "NSCLC not otherwise specified") were reduced from $24 \%$ to $13 \%$ of the cases, both sexes combined.

\section{Stages}

For both sexes, the fraction of patients with metastatic disease decreased during the 15-year period, from $57 \%$ to $46 \%$ for females and $52 \%$ to $45 \%$ for males. In parallel there was an increase in the fraction diagnosed with "localised disease" (stage I), from 17\% for both sexes in 2000 to $24 \%$ in females and $20 \%$ in males in 2016 (Fig 3). The fraction of patients with no information of stage at time of diagnoses was $11 \%$ for both sexes, with no change during the study period, this fraction is excluded in the calculations.

There were certain differences in stage distribution among the histological entities, in that distant metastases were most common in small-cell carcinomas, and more common in adenocarcinomas than in squamous cell carcinomas (Fig 3). For SCLC, metastatic disease was found in $60 \%$ of cases in both females and males, and less than $10 \%$ had localised disease. Metastases were present in approximately $50 \%$ of males with adenocarcinomas, and around $40 \%$ of females, while $25-30 \%$ of patients with squamous cell 
carcinomas had metastatic disease. Regional disease was the most frequent disease stage in squamous cell carcinomas. The fraction of localised disease has been relatively stable for all subtypes and both sexes throughout the period, encompassing a forth of females, and a fifth of males (Fig 3).

\section{Survival}

There was a large and clinically relevant survival improvement for the total cohort. Median survival for females increased with 120\% from 6.5 months in 2000 to 14.3 in 2015 (latest calculable number). Also for males, the median survival more than doubled from 5.4 to 11.4 months in 2016 (Fig 10). The improvement in median survival for patients with metastatic disease was, however, limited, increasing from 3.6 to 5.1 months (42\%) for females and from 3.2 to 4.2 months (31\%) for males (Fig 4A).

One-, 2- and 5-year relative survival for all stages combined improved from 2000 to 2016. The 1-year survival increased from $34 \%$ to $53 \%$ for females, and from $32 \%$ to $47 \%$ for males; the 2 -year survival from $23 \%$ to $40 \%$ for females and from $16 \%$ to $32 \%$ for males, while the 5 -year survival increased from 16 to $26 \%$ for females and from $9 \%$ to $22 \%$ for males (Fig 4B).

There was an improvement in relative survival which was most pronounced among patients with adenocarcinomas, but alsoevident in those with squamous cell carcinomasand for females with SCLC (Fig 5A). Overall, squamous cell carcinomas had better prognosis than adenocarcinomas among males throughout the period, and this seems to have become the case also for females during the last years.

There was an improvement in relative survival for all stages, but most pronounced for localised- and regional disease. Notably, there was a remarkable improvement from $26 \%$ to $64 \%$ in 5 -year survival among males with localised disease. In contrast, the 1-year survival in patients with metastatic disease improved from $13 \%$ and $14 \%$ in 2000 to $22 \%$ and $26 \%$ in 2016 for females and males, respectively (Fig 5B). 
Due to the substantial improvement in survival in localised disease, this patient group was studied in more detail. The prognosis in surgically treated males improved markedly, with 5-year relative survival increasing from approximately $50 \%$ at the beginning of the study period to more than $75 \%$ at the end. For surgically treated females, the 5 -year relative survival increased from approximately $75 \%$ to approximately $85 \%$. But the most profound improvement was found among patients who had not undergone surgery (Fig 6A). For non-surgically treated females, the 5 -year survival was below $25 \%$ until 2010, whereafter the curve points upwards, reaching more than $40 \%$ around 2016 . The 5 -year curves for males followed the same pattern, but fluctuated around 10\% until 2010, and reached $37 \%$ in 2016 .

The surgical rate among patients with localised disease varies with histological entity. Only $19 \%$ of SCLC patients underwent surgery, while the proportion was $72 \%$ among patients with adenocarcinomas (Fig 6B). Interestingly, the surgical rate for all subtypes with localised disease decreased with $3-6 \%$ from the first until the last part of the study period, but still, the 5-year survival for all patients with localised disease improved.

Very few SCLC patients underwent surgery even if they had localised disease. To be able to compare an appropriate number of patients, 2003-2009 was defined as the first period in Figure 6B, still counting only 28 SCLC patients. The difference in tumour biology between SCLC and other subtypes is reflected by the dismal 5-year survival of $24 \%$ in males with localised SCLC (Fig 6C).

Virtually no patients were offered SBRT until 2008, but the number has increased substantially since then. In 2016, $12 \%$ of all patients with lung cancer were treated with SBRT, and more than one third of patients with lung cancer are now offered curative treatment with either surgery or SBRT (data not shown). 


\section{Discussion}

In this study, incidence and survival of lung cancer at the national level in Norway from 2000 through 2016 was investigated, based on a sample size of over 44,000 patients. The mean age of patients with lung cancer has increased, adenocarcinoma has become the most common subtype, a larger proportion of patients received a histological diagnosis, and there has been a moderate shift in disease stage at diagnosis; more patients are now diagnosed with localised or regional disease. Most notably, there has been a significant improvement in both 1-, 2- and 5-year for both sexes, and for all subtypes and stages of disease, except for SCLC among males. The median survival increased from around 6 months for both sexes to 12 months for males, and to well above 12 months for females. Relative 5 -year survival was $26 \%$ for females and $22 \%$ for males in 2016 , which is higher than in other reports that we are aware of $[3,17$, 18]. The superior outcome for females compared to males has become more pronounced than in former reports [5].

There are several possible explanations for the substantial gain in survival. More frequent use of CTscans may have increased the number of incidentally detected non-symptomatic lung cancers, which may explain the migration to lower stages, though formal screening has not been implemented (ref). However, the change in fraction of patients with localised disease was small, with an increase from $17 \%$ to $24 \%$ among females, and from $17 \%$ to $20 \%$ among males, and the increase may also partly be explained by the adaptation of more accurate staging procedures such as PET-CT and endobronchial ultra sound (EBUS). The proportion of patients with localised disease undergoing surgery has decreased somewhat during the study period, but it is conceivable that patients are now more correctly selected for surgery.

The increased overall survival in patients with localised disease has also been seen in other recently published materials. In an analysis of the Veterans Administration Central Cancer Registry, where the 4- 
year survival increased from 39\% in 2001 to $53 \%$ in 2010 [19], compared to a 5 year-survival increase from 38 to $66 \%$ from 2000 to 2016 in our material. The largest survival change probably concerns patients with localised disease ineligible for surgery [19]. A substantial proportion of these patients are now offered SBRT [20, 21], accounting for 12\% of all newly diagnosed patients with lung cancer in 2016. By the end of the study period, more than a third of patients with lung cancer were treated with either SBRT or surgery, similar to what has recently been reported by others [22].

More emphasis on histological subtyping, including immunohistochemistry and genetic analyses, has undoubtedly contributed to a more correct stratification for targeted therapy, leading to an improved prognosis . EGFR mutation testing of all non-squamous NSCLC in conjunction with EGFR-directed therapy were implemented nation-wide in 2010, whereas ALK reflex testing and accompanying therapy were implemented from 2013 [10, 23].

Platinum-based chemotherapy was introduced around year 2000 [7, 24], which was relatively late compared to other countries, and second line therapies (docetaxel followed by pemetrexed) were introduced almost simultaneously [25]. Thus, there has been no change in the use of chemotherapy throughout the period, and the efficacy of such treatment is modest $[26,27]$. Notably, very few patients were treated with immunotherapy until 2016, when reimbursement for immunotherapy in PD-L1positive patients who relapsed after first line therapy was implemented [28-30].

The incidence of squamous cell carcinoma has been relatively stable over the period, whereas the incidence of adenocarcinoma has increased, especially among females. The relative incidence shift from squamous cell carcinoma to adenocarcinoma may be explained by differences in smoking habits, as usage of non-filtered cigarettes has decreased substantially over the latest decades [4, 31]. The stage distribution in these two subtypes are strikingly different, in that a much higher fraction of adenocarcinomas presented with metastatic disease. Still, the prognosis for the two groups, all stages 
combined, were fairly similar, with adenocarcinomas having the best outcomes for both sexes at the end of the period. One reason may be the efficacy of EGFR and ALK tyrosine kinase inhibitors which target mutations not present in squamous cell carcinomas.

Another reason for overall improved prognosis may be related to smoking. Patients with lung cancer who smoke have a worse overall prognosis than former-smokers [32]. Unfortunately, smoking among patients with cancer is not recorded by the CRN because of strict enforcement of personal secrecy regulations. The decline in daily smoking reported by Statistics Norway has been most pronounced in younger age groups, and was only modest around the mean age of lung cancer diagnosis. In the age group $65-74$, the proportion of daily smokers declined in the study period (2000-2016) from $24 \%$ to $15 \%$ in men, and from $18 \%$ to $12 \%$ in women (ssb.no). No smoking data exist for the age group $75+$, although they contribute one third of all incident lung cancers.

The prognosis of SCLC was relatively unchanged during the study period compared with the other histologic subtypes. There was almost no change in stage distribution, $60-70 \%$ are still diagnosed with metastatic disease, and less than $10 \%$ are diagnosed with localised disease. Furthermore, less than one in five with localised SCLC undergo surgery. There was a slight improvement in short-term prognosis among females, probably due to the introduction of a more structured trajectory for diagnosis and treatment through the conduct of a nation-wide randomised study of thoracic radiotherapy in limited disease SCLC [8].

Moreover, the implementation and refinement of national guidelines on diagnosis, treatment, and follow-up of lung cancer may have contributed to better management of this patient group [33]. All the mentioned factors may contribute to the improved prognosis.

A limitation of the study is the lack of details on stage and lack of other prognostic factors such as performance status, comorbidity and systemic treatment. In example the condensed staging system 
"Localised", "Regional" or "Metastatic" reduces the granularity of the analyses, but on the other hand this may lessen the effect of changes in the TNM-system during the period under study. Furthermore, the period approach may underestimate the true long-term survival in recent years, due to a carry-overeffect of data from previous years. Strengths of the study are the large sample size, high completeness of data and health care services being provided quite equally to patients in the country.

In conclusion, there have been notable changes in incidence patterns and a remarkable improvement in outcome for lung cancer over the last 16 years. The improvement for patients with metastatic disease has been relatively limited, but hopefully this subgroup of patients will have significantly bettered outcomes as the era of immunotherapy is entered.

\section{Acknowledgements}

Funding assistance was given by the South-Eastern Norway Regional Health Authority. The authors report no conflicts of interest. 


\section{References}

1. Brustugun, O.T., B. Møller, and A. Helland, Years of life lost as a measure of cancer burden on a national level. Br J Cancer, 2014. 111(5): p. 1014-20.

2. Torre, L.A., et al., Global cancer statistics, 2012. CA Cancer J Clin, 2015. 65(2): p. 87-108.

3. Allemani, C., et al., Global surveillance of trends in cancer survival 2000-14 (CONCORD-3): analysis of individual records for 37513025 patients diagnosed with one of 18 cancers from 322 population-based registries in 71 countries. Lancet, 2018.

4. Burns, D.M., C.M. Anderson, and N. Gray, Do changes in cigarette design influence the rise in adenocarcinoma of the lung? Cancer Causes Control, 2011. 22(1): p. 13-22.

5. Sagerup, C.M., et al., Sex-specific trends in lung cancer incidence and survival: a population study of 40,118 cases. Thorax, 2011. 66(4): p. 301-7.

6. Schwarzkopf, L., et al., Cost-components of lung cancer care within the first three years after initial diagnosis in context of different treatment regimens. Lung Cancer, 2015. 90(2): p. 274-80.

7. von Plessen, C., et al., Effectiveness of third-generation chemotherapy on the survival of patients with advanced non-small cell lung cancer in Norway: a national study. Thorax, 2008. 63(10): p. 866-871.

8. Grønberg, B.H., et al., Randomized phase II trial comparing twice daily hyperfractionated with once daily hypofractionated thoracic radiotherapy in limited disease small cell lung cancer. Acta Oncol, 2016. 55(5): p. 591-7.

9. Nyman, J., et al., SPACE - A randomized study of SBRT vs conventional fractionated radiotherapy in medically inoperable stage I NSCLC. Radiother Oncol, 2016. 121(1): p. 1-8.

10. Brustugun, O.T., et al., Mutasjonstesting ved ikke-småcellet lungekreft. Tidsskr Nor Laegeforen, 2012. 132(8): p. 952-5.

11. Brustugun, O.T., M. Sprauten, and Å. Helland, Real-world data on nivolumab treatment of nonsmall cell lung cancer. Acta Oncol, 2017. 56(3): p. 438-440.

12. Nilssen, Y., et al., Lung cancer treatment is influenced by income, education, age and place of residence in a country with universal health coverage. Int J Cancer, 2016. 138(6): p. 1350-60.

13. Nilssen, Y., et al., Lung cancer survival in Norway, 1997-2011: from nihilism to optimism. Eur Respir J, 2016. 47(1): p. 275-87.

14. Larsen, I.K., et al., Data quality at the Cancer Registry of Norway: an overview of comparability, completeness, validity and timeliness. Eur J Cancer, 2009. 45(7): p. 1218-31.

15. Perme, M.P., J. Stare, and J. Estève, On estimation in relative survival. Biometrics, 2012. 68(1): $\mathrm{p}$. 113-20.

16. Brenner, H. and B. Rachet, Hybrid analysis for up-to-date long-term survival rates in cancer registries with delayed recording of incident cases. Eur J Cancer, 2004. 40(16): p. 2494-501.

17. Francisci, S., et al., Survival patterns in lung and pleural cancer in Europe 1999-2007: Results from the EUROCARE-5 study. Eur J Cancer, 2015.

18. Driessen, E.J., et al., Trends in treatment and relative survival among Non-Small Cell Lung Cancer patients in the Netherlands (1990-2014): Disparities between younger and older patients. Lung Cancer, 2017. 108: p. 198-204.

19. Boyer, M.J., et al., Improved Survival of Stage I Non-Small Cell Lung Cancer: A VA Central Cancer Registry Analysis. J Thorac Oncol, 2017. 12(12): p. 1814-1823. 
20. Palma, D., et al., Impact of introducing stereotactic lung radiotherapy for elderly patients with stage I non-small-cell lung cancer: a population-based time-trend analysis. J Clin Oncol, 2010. 28(35): p. 5153-9.

21. McMurry, T.L., et al., Treatment of stage I non-small cell lung cancer: What's trending? J Thorac Cardiovasc Surg, 2017.

22. Kapadia, N.S., et al., Patterns of Treatment and Outcomes for Definitive Therapy of Early Stage Non-Small Cell Lung Cancer. Ann Thorac Surg, 2017. 104(6): p. 1881-1888.

23. Berg, J., et al., EGFR mutation testing of lung cancer patients - Experiences from Vestfold Hospital Trust. Acta Oncol, 2016. 55(2): p. 149-55.

24. von Plessen, C., et al., Palliative chemotherapy beyond three courses conveys no survival or consistent quality-of-life benefits in advanced non-small-cell lung cancer. Br J Cancer, 2006. 95(8): p. 966-73.

25. Helbekkmo, N., et al., Vinorelbine/carboplatin vs gemcitabine/carboplatin in advanced NSCLC shows similar efficacy, but different impact of toxicity. Br J Cancer, 2007. 97(3): p. 283-9.

26. Davies, J., et al., Real-world treatment patterns for patients receiving second-line and third-line treatment for advanced non-small cell lung cancer: A systematic review of recently published studies. PLoS One, 2017. 12(4): p. e0175679.

27. Schiller, J.H., et al., Comparison of four chemotherapy regimens for advanced non-small-cell lung cancer. N Engl J Med, 2002. 346(2): p. 92-98.

28. Herbst, R.S., et al., Pembrolizumab versus docetaxel for previously treated, PD-L1-positive, advanced non-small-cell lung cancer (KEYNOTE-010): a randomised controlled trial. Lancet, 2016. 387(10027): p. 1540-50.

29. Borghaei, H., et al., Nivolumab versus Docetaxel in Advanced Nonsquamous Non-Small-Cell Lung Cancer. N Engl J Med, 2015. 373(17): p. 1627-39.

30. Brahmer, J., et al., Nivolumab versus Docetaxel in Advanced Squamous-Cell Non-Small-Cell Lung Cancer. N Engl J Med, 2015. 373(2): p. 123-35.

31. Skretting, A., et al., Rusmidler i Norge 2016. , in Statistikk 2016. 2017, Norwegian Institute of Public Health: Oslo.

32. Koshiaris, C., et al., Smoking cessation and survival in lung, upper aero-digestive tract and bladder cancer: cohort study. Br J Cancer, 2017. 117(8): p. 1224-1232.

33. Christensen, N.L., et al., Lung cancer guidelines in Sweden, Denmark, Norway and Finland: a comparison. Acta Oncol, 2017. 56(7): p. 943-948. 


\section{Figure legends}

Figure 1A The incidence of lung cancer by sex and year of observation. 1B. Prevalence of lung cancer by time after diagnosis and observation year. 1C. Mean age at diagnosis of lung cancer by sex and observation year.

Figure 2. Distribution of incident lung cancer according to histological subtype, by sex and year of observation.

Figure 3. Distribution of lung cancers according to stage by histological subtype, sex and year of observation. Patients registered with unknown stage (11\% of cases) are excluded.

Figure 4A. Median survival of patients with lung cancer by sex, for patients with metastatic disease, and all stages. 4B. Relative survival (1-year, 2-year and 5-year) among patients with lung cancer by sex and year of observation.

Figure 5A. Relative survival (1-year, 2-year and 5-year) for patients with lung cancer by histological subgroup, sex and year of observation. 5B. Relative survival of patients with lung cancer by stage, sex and year of observation.

Figure $6 \mathrm{~A}$ Overall survival of patients with localised lung cancer 1,2 , and 5 years after diagnosis, by calendar year of observation, treatment and sex.6B. Percent operated among patients with lung cancer diagnosed with localised disease, by histological subgroup and period of diagnosis. 6C. Survival of patients with localised lung cancer, by histological subgroup and period of observation. 